\title{
THE STRONG MAXIMAL FUNCTION ON A NILPOTENT GROUP
}

\author{
MICHAEL CHRIST
}

\begin{abstract}
An analogue of the strong maximal function of Jessen, Marcinkiewicz, and Zygmund is shown to be bounded on $L^{p}$, for all $p>1$, on a nilpotent Lie group.
\end{abstract}

\section{INTRODUCTION}

This is one of a series of papers [C1], [C2] concerned with non-translationinvariant singular integral operators and maximal functions. Operators which are left-invariant with respect to some nilpotent group structure on $\mathbb{R}^{n}$ provide natural models for substantial classes of such objects. In particular, the study of such operators in [C1] was a preliminary step in the treatment of a large, diffeomorphism - rather than group-invariant class of singular averages in [C2]. Moreover, these invariant examples are of interest in their own right.

The strong maximal function on $\mathbb{R}^{n}$ is defined by

$$
M_{\text {strong }} f(x)=\sup _{r \in\left(\mathbb{R}^{+}\right)^{n}} \int_{|y| \leq 1}\left|f\left(x-\delta_{r} y\right)\right| d y,
$$

where $r=\left(r_{1}, \ldots, r_{n}\right)$ and $\delta_{r} y=\left(r_{1} y_{1}, \ldots, r_{n} y_{n}\right)$. It is a classical result of Jessen, Marcinkiewicz, and Zygmund [JMZ] that for each $p>1, M_{\text {strong }}$ is bounded on $L^{p}$. In the present article we establish a generalization to the setting of nilpotent Lie groups.

In $\mathbb{R}^{n}$ consider for each $1 \leq j \leq n$ the maximal function

$$
M_{j} f(x)=\sup _{t \in \mathbb{R}^{+}} \int_{|s| \leq 1, s \in \mathbb{R}}|f(x-(0, \ldots, 0, t s, 0, \ldots, 0))| d s,
$$

with the entry $t s$ in the $j$ th place. Then there is a pointwise bound

$$
M_{\text {strong }} f(x) \leq C M_{1} M_{2} \cdots M_{n} f(x) .
$$

Moreover, it follows at once from the boundedness of the Hardy-Littlewood maximal function on $\mathbb{R}^{1}$ that each $M_{j}$ is bounded on $L^{p}\left(\mathbb{R}^{n}\right)$, for all $p>1$. Hence $M_{\text {strong }}$ is bounded.

Received by the editors February 1, 1988.

1980 Mathematics Subject Classification (1985 Revision). Primary 42B25, 43A15.

Key words and phrases. Hardy-Littlewood maximal function, strong maximal function, nilpotent group.

Research supported by the National Science Foundation and the Australian National University. 
This argument fails to generalize directly to nonabelian nilpotent groups. Consider the Heisenberg group $\mathbb{H}^{1}$. It may be identified with $\mathbb{R}^{3}$, with group law

$$
\left(x_{1}, x_{2}, x_{3}\right) \cdot\left(x_{1}^{\prime}, x_{2}^{\prime}, x_{3}^{\prime}\right)=\left(x_{1}+x_{1}^{\prime}, x_{2}+x_{2}^{\prime}, x_{3}+x_{3}^{\prime}+2\left(x_{2} x_{1}^{\prime}-x_{1} x_{2}^{\prime}\right)\right) .
$$

Set $\delta_{r} x=\left(r_{1} x_{1}, r_{2} x_{2}, r_{3} x_{3}\right)$ and observe that $\delta_{r}$ is a group automorphism of $\mathbb{H}^{1}$ only when $r_{3}=r_{1} r_{2}$. Our strong maximal function on $\mathbb{H}^{1}$ is defined by

$$
M_{\text {strong }} f(x)=\sup _{r \in\left(\mathbb{R}^{+}\right)^{3}} \int_{|y| \leq 1}\left|f\left(x \cdot\left(\delta_{r} y\right)^{-1}\right)\right| d y .
$$

This operator is invariant under left translation. Define also

$$
M_{3} f(x)=\sup _{t \in \mathbb{R}^{+}} \int_{|s| \leq 1, s \in \mathbb{R}}\left|f\left(x \cdot(0,0, t s)^{-1}\right)\right| d s
$$

and

$$
M_{\text {sing }} f(x)=\sup _{r \in\left(\mathbb{R}^{+}\right)^{2}} \int_{|u| \leq 1, u \in \mathbb{R}^{2}}\left|f\left(x \cdot\left(r_{1} u_{1}, r_{2} u_{2}, 0\right)^{-1}\right)\right| d u .
$$

Then pointwise $M_{\text {strong }} f(x) \leq C M_{3} M_{\text {sing }} f(x)$. Both $M_{\text {sing }}$ and $M_{3}$ are defined by dilations $\delta_{r}$ which are automorphisms of the $\mathbb{H}^{1}$ group structure. Again it follows at once from the one-dimensional result that $M_{3}$ is bounded on $L^{p}\left(\mathbb{H}^{1}\right)$ for all $p>1$, and so boundedness of $M_{\text {strong }}$ would follow from boundedness of $M_{\text {sing }}$. Moreover by taking $f$ to be continuous but otherwise arbitrary and letting $r_{3} \rightarrow 0$ in the definition of $M_{\text {strong }}$, we find that the boundedness of $M_{\text {sing }}$ is completely equivalent to that of $M_{\text {strong }}$. However there is no simple majorization of the type $M_{\text {sing }} \leq M_{1} M_{2}$, essentially because the submanifold $\left\{x: x_{3}=0\right\}$ is not a subgroup of $\mathbb{H}^{1}$.

Define a simpler maximal function $M_{\text {better }}$ on $\mathbb{H}^{1}$ by restricting $r_{1}=r_{2}$ in the supremum in the definition of $M_{\text {sing }}$. $M_{\text {better }}$ is still given by averaging over lower-dimensional sets, but is simpler than $M_{\text {sing }}$ because it involves a supremum only over a single parameter. It is a nonabelian analogue of the maximal function in $\mathbb{R}^{3}$ defined by

$$
M_{\text {paraboloid }} f(x)=\sup _{t>0} \int_{|u| \leq 1, u \in \mathbb{R}^{2}}\left|f\left(x_{1}-t u_{1}, x_{2}-t u_{2}, x_{3}-t^{2}\left(u_{1}^{2}+u_{2}^{2}\right)\right)\right| d u
$$

with the usual Euclidean group structure on $\mathbb{R}^{3}$. The $L^{p}$ boundedness of $M_{\text {paraboloid }}$ follows from the method of [SW], which relied on the Fourier transform. $M_{\text {better }}$ was treated later by Phong and Stein [PS1, PS2]; the Euclidean Fourier transform could no longer be directly applied since the Heisenberg group is not abelian.

Our study of the strong maximal function is based on the technique developed in [C1, C2], [RiS1, RiS2]. Two modifications of a technical nature will be introduced here to adapt the method to multiple-parameter problems.

\section{Statement OF Results}

Let $\mathbf{g}$ denote a nilpotent Lie algebra of dimension $d$ and let $\left\{X_{j}: 1 \leq\right.$ $j \leq d\}$ be a basis for $\mathbf{g}$ as a vector space. Let $G$ be the unique connected, simply connected Lie group with Lie algebra $\mathbf{g}$, identify each $X_{j}$ with the corresponding left-invariant vector field on $G$, and identify $G$ with $\mathbf{g}$ via the 
exponential map exp: $\mathbf{g} \mapsto G$ based at the group identity element, denoted by 0 . This identifies $G$ with $\mathbb{R}^{d}$ under the coordinate system $\left(x_{1}, \ldots, x_{d}\right) \in$ $\mathbb{R}^{d} \leftrightarrow \exp \left(\sum x_{j} X_{j}\right) \in G$. Haar measure on $G$ is simply Lebesgue measure in these coordinates, and $L^{p}$ will always be defined with respect to Haar measure. Write $\delta_{r} x=\left(r_{1} x_{1}, \ldots, r_{d} x_{d}\right)$. Throughout the paper $\mathbb{R}^{+}$denotes $(0, \infty)$ and $\mathbb{N}=\{0,1,2, \ldots\}$.

Define

$$
M_{\text {strong }} f(x)=\sup _{r \in\left(\mathbb{R}^{+}\right)^{d}} \int_{|y| \leq 1}\left|f\left(x \cdot\left(\delta_{r} y\right)^{-1}\right)\right| d y .
$$

$M f$ is measurable for each $f \in L_{\text {loc }}^{1}$, since $r$ may be restricted to a countable dense set. It is a generalization to $G$ of the strong maximal function. Our main result is then

Theorem 2.1. $M_{\text {strong }}$ is bounded on $L^{p}(G)$ for all $p>1$.

On $\mathbb{H}^{1}$ we have already seen that the boundedness of $M$ may be reduced to that of an operator which is more singular, but which is defined by a group of dilations which act as automorphisms of the group structure. The same may be achieved in general, by passing to a higher-dimensional group as in [RiS1, RiS2, $\mathrm{C} 2]$ (in the latter one passes not to a group, but to a more general setting).

To do this fix an integer $s$ such that all commutators of length greater than $s$ in $\mathbf{g}$ are zero. Let $\mathbf{h}$ denote the free nilpotent Lie algebra of step $s$ on $d$ generators $Z_{1}, \ldots, Z_{d}$. Let $H=\exp (\mathbf{h})$ be the associated connected, simply connected nilpotent Lie group, and let $D$ be the dimension of $\mathbf{h}$ as a vector space. On $H$ define

$$
M_{H} f(x)=\sup _{r \in\left(\mathbb{R}^{+}\right)^{d}} \int_{u \in \mathbb{R}^{d},|u| \leq 1}\left|f\left(x \cdot \exp \left[-\sum r_{j} u_{j} Z_{j}\right]\right)\right| d u .
$$

There is a natural dilation structure on $H$, since for each $r \in\left(\mathbb{R}^{+}\right)^{d}$ there exists a unique Lie algebra automorphism $\delta_{r}: \mathbf{h} \mapsto \mathbf{h}$ satisfying $\delta_{r}\left(Z_{j}\right)=r_{j} Z_{j}$ for each $j$. Via the exponential map $\left\{\delta_{r}\right\}$ goes over to a (multiplicative) group of automorphisms of $H$, which we also denote by $\left\{\delta_{r}\right\}$. Then $M_{H}$ is invariant under both left translation on $H$ and the dilations $\delta_{r}$.

The next lemma reduces matters to the study of $M_{H}$. It plays a fundamental role in the work of Ricci and Stein [RiS1, RiS2], who have emphasized how various results concerning nilpotent groups equipped with groups of dilations which are group automorphisms extend to the nonautomorphic case.

Lemma 2.2. There exists a bijective diffeomorphism $\Phi: H \mapsto \mathbb{R}^{D}$ which preserves Lebesgue measure such that if $\pi: \mathbb{R}^{D} \mapsto \mathbb{R}^{d}=G$ is defined by forgetting the last $D-d$ coordinates, then $\pi \Phi\left(\exp \left(\sum u_{j} Z_{j}\right)(x)\right) \equiv \exp \left(\sum u_{j} X_{j}\right)(\pi \Phi(x))$ for all $x \in H$ and $u \in \mathbb{R}^{d}$.

For the proof [RiS1, RiS2] it suffices to take $\pi$ to be the quotient map from $H$ onto $G$ in appropriate coordinates. Suppose $M_{H}$ were known to be bounded on some $L^{p}(H)$. In $\mathbb{R}^{D}$ write $x=\left(\pi x, x^{\prime}\right)$. Given a compactly supported $f \in$ $L^{p}(G)$, consider for each $N>0$ the function $F_{N}(x)=f(\pi x) \chi_{N}(x)$, where $\chi_{N}$ denotes the characteristic function of the set $\left\{x \in \mathbb{R}^{D}:\left|x^{\prime}\right| \leq N\right\}$. There exists an exponent $w$ such that for any compact region $K \subset G$, for all sufficiently large $N,\left\|M_{H} F_{N}\right\|_{L^{p}(H)} \geq C N^{w}\left\|M_{\text {strong }} f\right\|_{L^{p}(K)}$ and $\left\|F_{N}\right\|_{L^{p}} \sim N^{w}\|f\|_{L^{p}}$. In 
fact for large $N$ there is a pointwise bound $M_{H} F_{N}(x) \geq M_{\text {strong }} f(\pi x)$ for all $x$ satisfying $\pi x \in K$ and $\left|x^{\prime}\right| \leq N / 2$. Thus $M_{\text {strong }}$ would be bounded on $L^{p}(G)$. This lifting technique originates in [RoS], in a more general form, and was applied, in disguise, to maximal functions and Hilbert transforms along curves in [C2]. Its applicability in closely related contexts was pointed out to this author by A. Nagel and N. Varopoulos.

It is helpful to make a further reduction, to a discretized version of $M_{H}$. Introduce a nonnegative auxiliary function $a \in C_{0}^{\infty}\left(\mathbb{R}^{d}\right)$, identically one on $\left\{u: 1 / 2 \leq u_{i} \leq 1\right.$ for all $\left.i\right\}$ and supported on $\left\{u: 1 / 4 \leq u_{i} \leq 2\right.$ for all $\left.i\right\}$. For each $J \in \mathbb{Z}^{d}$ let $\mu_{J}$ be the measure on $H$ defined by

$$
\int f d \mu_{J}=\int f\left(\exp \left(\sum 2^{-J_{j}} u_{j} Z_{j}\right)\right) a(u) d u .
$$

In the definition of $M_{H}$ it suffices to replace the region of integration $\{|u| \leq 1\}$ by $\left\{u: 0 \leq u_{i} \leq 1\right.$ for all $\left.i\right\}$, for the old $M_{H}$ is majorized by $2^{d}$ of the new ones; by symmetry the boundedness of one implies boundedness of all $2^{d}$ of them. Then there is the further majorization

$$
M_{H} f(x) \leq C \sup _{J}\left|f * \mu_{J}\right|
$$

for all nonnegative $f \in L_{\text {loc }}^{1}(H)$.

We have seen how the strong maximal function is inextricably linked with multiple-parameter maximal functions along surfaces. It is therefore natural to consider such operators from the outset, rather than merely in the free, homogeneous setting of $M_{H}$. The next result subsumes Theorem 2.1.

Theorem 2.3. Let $d \in \mathbb{Z}^{+}$, let $G$ be a connected, simply connected nilpotent Lie group of dimension $d$, and let $A$ be a finite subset of $\mathbb{N}^{d} \backslash\{(0,0, \ldots, 0)\}$. For each $\alpha \in A$ let $X_{\alpha}$ be an element of the Lie algebra of $G$. Consider the maximal function

$$
M f(x)=\sup _{r \in\left(\mathbb{R}^{+}\right)^{d}} \int_{u \in \mathbb{R}^{d},|u| \leq 1}\left|f\left(x \cdot \exp \left(-\sum_{\alpha \in A} r^{\alpha} u^{\alpha} X_{\alpha}\right)\right)\right| d u,
$$

where $r^{\alpha}=\prod_{1 \leq i \leq d} r_{i}^{\alpha_{i}}$ and $u^{\alpha}=\prod u_{i}^{\alpha_{i}}$. Then $M$ is bounded on $L^{p}(G)$ for all $p>1$.

The next result is stronger yet. Let $A$ be a finite subset of $\mathbb{N}^{d} \backslash\{(0,0, \ldots, 0)\}$ and let $G$ be the connected, simply connected nilpotent Lie group whose Lie algebra is free of some step $s$ on generators $\left\{X_{\alpha}: \alpha \in A\right\}$. For $r \in\left(\mathbb{R}^{+}\right)^{|A|}$, let $\delta_{r}: G \mapsto G$ denote the unique automorphism satisfying $\delta_{r} \exp \left(X_{\alpha}\right)=\exp \left(r_{\alpha} X_{\alpha}\right)$ for each $\alpha$, where $r_{\alpha}$ are the components of $r$. Let $a \in C_{0}^{\infty}\left(\mathbb{R}^{d}\right)$ be as above. For each $J \in \mathbb{Z}^{|A|}$ define a finite measure $\mu_{J}$ on $G$ by

$$
\begin{aligned}
\int f d \mu_{J} & =\int_{\mathbb{R}^{d}} f\left(\delta_{2^{-J}} \exp \left(\sum u^{\alpha} X_{\alpha}\right)\right) a(u) d u \\
& =\int f\left(\exp \left(\sum 2^{-J_{\alpha}} u^{\alpha} X_{\alpha}\right)\right) a(u) d u
\end{aligned}
$$

Here $2^{-J}$ is the $|A|$-tuple $\left(2^{-J_{\alpha}}: \alpha \in A\right)$. 
Theorem 2.4. The maximal function

$$
M f(x)=\sup _{J \in \mathbb{N}|A|}\left|f * \mu_{J}\right|
$$

is bounded on $L^{p}(G)$ for all $p>1$.

Here it is essential that the dilation group should be discrete, for if we consider the dilates of $\mu_{0}$ by all $r \in\left(\mathbb{R}^{+}\right)^{|A|}$ and take the supremum over all such $r$ then the resulting maximal function need not be bounded on all $L^{p}, p>1$. In fact operators of the same general type as the maximal spherical averages in $\mathbb{R}^{n}[\mathrm{~B}, \mathrm{SW}]$ would be of this class.

To deduce Theorem 2.3 from Theorem 2.4 apply the lifting Lemma 2.2 to reduce matters to the case where $G$ is the free nilpotent group of appropriate step whose Lie algebra is generated by $\left\{X_{\alpha}\right\}$. As remarked before the statement of Theorem 2.3, it suffices to discretize the dilation group. For $\rho \in\left(\mathbb{R}^{+}\right)^{d}$ define dilations $\tilde{\delta}_{\rho}(x)=\delta_{r}(x)$, where $r_{\alpha}=\rho^{\alpha}$. Then $\left\{\tilde{\delta}_{\rho}: \rho \in\left(\mathbb{R}^{+}\right)^{d}\right\}$ is a subgroup of $\left\{\delta_{r}\right\} . M$ is dominated pointwise, for nonnegative $f$, by the variant obtained by replacing the dilations $\left\{\delta_{r}: r=2^{J}, J \in \mathbb{Z}^{|A|}\right\}$ by $\left\{\tilde{\delta}_{\rho}: \rho=\right.$ $\left.2^{I}, I \in \mathbb{Z}^{d}\right\}$ in its definition.

In Theorem 2.3 it is not assumed that the $X_{\alpha}$ are linearly independent or even distinct. Thus the surface $\left\{\exp \left(\sum u^{\alpha} X_{\alpha}\right): u \in \mathbb{R}^{d}\right\}$ is simply the image of $\mathbb{R}^{d}$ under any function $F: \mathbb{R}^{d} \mapsto G$ which is a polynomial in exponential coordinates on $G$ and maps 0 to itself.

The remainder of the paper is devoted to the proof of Theorem 2.4. I am grateful to P. Sjögren and M. Cowling for helpful comments and to A. Nagel and S. Wainger for stimulating conversations. Related results for the Heisenberg group have been obtained by Ricci and Sjögren [RSj2].

\section{DECOMPOSITION OF THE OPERATOR}

We use the following notation: $A$ is a finite subset of $\mathbb{N}^{d} \backslash\{0\}, \mathbf{g}$ is the free nilpotent Lie algebra of some step $s$ on generators $\left\{X_{\alpha}: \alpha \in A\right\}, G$ is the associated connected, simply connected nilpotent group, and exp: $\mathbf{g} \mapsto G$ is the exponential map, based at 0 , the group identity element. For each $r \in$ $\left(\mathbb{R}^{+}\right)^{|A|}, \delta_{r}$ is the unique automorphism of $\mathbf{g}$ satisfying $\delta_{r} X_{\alpha}=r_{\alpha} X_{\alpha}$ for all $\alpha$, where the $r_{\alpha}$ are the components of $r$. As earlier we also write $\delta_{r}$ for the corresponding group automorphism of $G$. For each $\beta \in\left(\mathbb{Z}^{+}\right)^{|A|} \backslash\{0\}$, let $\mathbf{g}_{\beta}=\left\{Y \in \mathbf{g}: \delta_{r} Y=r^{\beta} Y\right.$ for all $\left.r \in\left(\mathbb{R}^{+}\right)^{|A|}\right\}$, a linear subspace of $\mathbf{g}$. For each $\alpha \in A$ let $\mathbf{g}^{\alpha}$ be the direct sum of all $\mathbf{g}_{\beta}$ for which $\beta_{\alpha} \neq 0$. Let $d_{\alpha}$ be the dimension of $\mathbf{g}^{\alpha}$ as a vector space. For each $\beta$ fix a vector space basis $\left\{Y_{\beta, j}: 1 \leq j \leq \operatorname{dim}\left(\mathbf{g}_{\beta}\right)\right\}$ for $\mathbf{g}_{\beta}$. All but finitely many of the $\mathbf{g}_{\beta}$ equal $\{0\}$ and will be ignored. For each $\alpha,\left\{Y_{\beta, j}: \beta_{\alpha} \neq 0\right\}$ is a basis for $\mathbf{g}^{\alpha}$. This establishes an identification of $\mathbf{g}^{\alpha}$ with $\mathbb{R}^{d_{\alpha}}$.

Fix $b_{\alpha} \in C_{0}^{\infty}\left(\mathbf{g}^{\alpha}\right)$ satisfying $\int b_{\alpha}=1$. For $k \in \mathbb{N}^{+}$set $b_{\alpha, k}(x)=2^{k d_{\alpha}} b_{\alpha}\left(2^{k} x\right)$, where $2^{k} x=\left(2^{k} x_{1}, 2^{k} x_{2}, \ldots, 2^{k} x_{d_{\alpha}}\right)$; these dilations will in general not be automorphisms of $\mathbf{g}^{\alpha}$. Define measures $\sigma_{\alpha, k}$ and $\lambda_{\alpha, k}$ on $G$ by

$$
\sigma_{\alpha, k}=\exp _{*}\left(b_{\alpha, k}(u) d u\right),
$$

where exp: $\mathbf{g}^{\alpha} \mapsto G$ is the restriction to $\mathbf{g}^{\alpha}$ of the exponential map for $\mathbf{g}$, 
$\exp _{*}(\cdot)$ denotes the push-forward of a measure, and

$$
\lambda_{\alpha, k}=\sigma_{\alpha, k}-\sigma_{\alpha, k-1} \quad \text { for } k \geq 1 .
$$

For $K \in \mathbb{Z}^{+|A|}$ define

$$
\Lambda^{K}=\prod_{\alpha \in A} \lambda_{\alpha, K_{\alpha}},
$$

where $\Pi$ denotes the convolution product of measures, taken according to an arbitrarily chosen linear ordering of $A$ which will henceforth remain fixed. For $J \in \mathbb{Z}^{|A|}$ define dilated measures $\Lambda_{J}^{K}$ by

$$
\int f d \Lambda_{J}^{K}=\int f\left(\delta_{r} x\right) d \Lambda^{K}(x), \quad r=2^{-J} .
$$

For each subset $E \subset A$ define

$$
\tau^{E}=\prod_{\alpha \in E} \sigma_{\alpha, 0}
$$

with the convolution product taken again in order of increasing $\alpha$, and define $\tau_{J}^{E}$ by

$$
\int f d \tau_{J}^{E}=\int f\left(\delta_{r} x\right) d \tau^{E}(x), \quad r=2^{-J} .
$$

Let $\delta$ denote the Dirac mass at $0 \in G$.

There is then the decomposition

$$
\delta=\prod_{\alpha \in A}\left[\left(\delta-\sigma_{\alpha, 0}\right)+\sigma_{\alpha, 0}=\prod_{\alpha \in A}\left(\delta-\sigma_{\alpha, 0}\right)+\sum_{\varnothing \neq E \subset A} c_{E} \tau^{E},\right.
$$

where each $c_{E}$ is an integer. Expanding $\delta-\sigma_{\alpha, 0}=\sum_{k=1}^{\infty} \lambda_{\alpha, k}$ for each $\alpha$ and then dilating gives for each $J \in \mathbb{Z}^{|A|}$

$$
\mu_{J}=\sum_{K} \mu_{J} * \Lambda_{J}^{K}+\sum_{E \neq \varnothing} c_{E} \mu_{J} * \tau_{J}^{E},
$$

where the sum is over all $K \in\left(\mathbb{Z}^{+}\right)^{|A|}$. This leads to a majorization of the maximal function $\sup \left|f * \mu_{J}\right|$ by an infinite sum of maximal functions, one for each $E$ and one for each $K$.

Lemma 3.1. For each $E \neq \varnothing$ and $p>1$ the maximal function

$$
\sup _{J}\left|f * \mu_{J} * \tau_{J}^{E}\right|
$$

is bounded on $L^{p}(G)$.

For the proof fix $E$. As a vector space split $\mathbf{g}$ as the direct sum $\mathbf{g}_{0} \oplus \mathbf{g}_{\infty}$, where $\mathbf{g}_{0}=\operatorname{span}\left\{\mathbf{g}^{\alpha}: \alpha \in E\right\}$ and $\mathbf{g}_{\infty}=\operatorname{span}\left\{\mathbf{g}_{\beta}: \beta_{\alpha}=0 \forall \alpha \in E\right\}$. Both are Lie subalgebras of $\mathbf{g}$. Moreover $\mathbf{g}_{0}$ is an ideal and $\mathbf{g}_{\infty}$ is nothing but the free nilpotent Lie algebra of step $s$ on $|A|-|E|$ generators. Let $G_{0}, G_{\infty}$ be the associated connected, simply connected groups, both regarded as subgroups of $G$. Each element of our basis $\left\{Y_{\beta, j}\right\}$ belongs to exactly one of the two subalgebras, so both inherit bases and hence the exponential map defines canonical coordinates on the $G_{i}$. Every element of $G$ admits a unique representation $x=w v$, where $w \in G_{\infty}$ and $v \in G_{0}$. Haar measure on $G$ is simply the product of the two Haar measures. 
Recall the map $u \mapsto \exp \left(\sum u^{\alpha} X_{\alpha}\right)$ from $\mathbb{R}^{d}$ to $G$ which traces out the surface on which $\mu_{0}$ is supported. Factor it as $F_{\infty}(u) \cdot F_{0}(u)$, where each $F_{i}$ : $\mathbb{R}^{d} \mapsto G_{i}$ is a polynomial in canonical coordinates. $G_{0}$ is a normal subgroup, whence

$$
F_{\infty}(u)=\exp \left(\sum_{\alpha \notin E} u^{\alpha} X_{\alpha}\right)
$$

although $F_{0}$ will in general not have such a simple form.

$\mu_{0}$ decomposes as

$$
\mu_{0}=\int_{\mathbb{R}^{d}} \delta_{F_{\infty}(u)} * \delta_{F_{0}(u)} a(u) d u,
$$

where $\delta_{x}$ denotes here the Dirac mass at $x \in G$. Thus

$$
\mu_{0} * \tau_{0}^{E}=\int \delta_{F_{\infty}(u)} *\left(\delta_{F_{0}(u)} * \tau_{0}^{E}\right) a(u) d u .
$$

Observe that because of the form of the group law, $\tau_{0}^{E}$ is absolutely continuous and even has a $C^{\infty}$ density with respect to Haar measure on $G_{0}$. So if on $G_{0}$ we let $\nu$ equal Lebesgue measure times the characteristic function of a sufficiently large ball, then $\delta_{F_{0}(u)} * \tau_{0}^{E} \leq C \nu$ for all $u \in \operatorname{support}(a)$. Hence

$$
\mu_{0} * \tau_{0}^{E} \leq C\left(\int \delta_{F_{\infty}(u)} a(u) d u\right) * \nu \leq C \tilde{\mu}_{0} * \nu,
$$

where $\tilde{\mu}_{0}$ is the measure $\left(F_{\infty}\right)_{*}(a(u) d u)$ supported on $G_{\infty} \subset G$, an analogue on $G_{\infty}$ of $\mu_{0}$.

Both subgroups $G_{0}, G_{\infty}$ are invariant under the dilation group $\left\{\delta_{r}\right\}$ since their Lie algebras are. We write $\delta_{r}$ also for the restriction to either. On $G_{0}$ consider the maximal function

$$
N f(x)=\sup _{r \in\left(\mathbb{R}^{+}\right)^{|A|}} \int_{y \in G_{0},|y| \leq 1}\left|f\left(x \cdot\left(\delta_{r} y\right)^{-1}\right)\right| d y .
$$

Since the $\delta_{r}$ are group automorphisms of $G_{0}$, the simple iteration argument described in the first two paragraphs of the introduction applies just as for the strong maximal function in $\mathbb{R}^{n}$ and establishes

Lemma 3.2. $N$ is bounded on $L^{p}\left(G_{0}\right)$ for all $p>1$.

For any continuous function $f$ defined on $G$ and for each $w \in G_{\infty}$ set $f^{w}(v)=f(w v)$, a function on $G_{0}$. Define

$$
N^{\prime} f(w v)=N f^{w}(v) .
$$

Since Haar measure on $G$ is the product measure, it follows from Fubini's theorem that $N^{\prime}$ is bounded on $L^{p}(G)$ for all $p>1$.

We now proceed by induction on $|A|$, the number of generators of the Lie algebra, hypothesizing that Theorem 2.4 is already known in the case of fewer generators. On $G_{\infty}$ define $\tilde{\mu}_{J}$ in terms of $\tilde{\mu}_{0}$ by dilating in the customary way, and consider the maximal function

$$
\widetilde{M} f(x)=\sup _{I}\left|f * \tilde{\mu}_{I}\right|,
$$


precisely the sort of operator with which we began on $G$. Since $\mathbf{g}_{\infty}$ is the free Lie algebra of step $s$ on $|A|-|E|$ generators, $\widetilde{M}$ is bounded on $L^{p}\left(G_{\infty}\right)$ for all $p>1$ by the inductive hypothesis. On $G$ define

$$
\widetilde{M}^{\prime} f(x)=\sup _{I}\left|f * \tilde{\mu}_{I}\right|,
$$

where $\tilde{\mu}_{I}$ denotes also the push-forward of $\tilde{\mu}_{I}$ to $G$ via the inclusion map. (To define $\widetilde{M}^{\prime}$ in terms of $\widetilde{M}$ as $N^{\prime}$ was defined in terms of $N$ would require writing elements of $G$ as $x=v w$ with $v \in G_{0}$ and $w \in G_{\infty}$ rather than in our usual order.) Then $\widetilde{M}^{\prime}$ is bounded on $L^{p}(G)$. For any continuous, nonnegative $f$ defined on $G$, pointwise and for all $J$

$$
\begin{aligned}
f *\left(\mu_{J} * \tau_{J}^{E}\right) & \leq C\left(f * \tilde{\mu}_{J}\right) * \nu_{J} \leq C\left(\sup _{I} f * \tilde{\mu}_{I}\right) * \nu_{J} \\
& \leq C\left(\widetilde{M^{\prime} f}\right) * \nu_{J} \leq C N^{\prime} \widetilde{M}^{\prime} f .
\end{aligned}
$$

The proof of Lemma 3.1 is complete.

\section{THE MAIN STEP}

This section is devoted to the proof of

Proposition 4.1. There exists $\varepsilon>0$ such that

$$
\left\|\sup _{J}\left|f * \mu_{J} * \Lambda_{J}^{K}\right|\right\|_{2} \leq C 2^{-\varepsilon|K|}\|f\|_{2}
$$

for all $f \in L^{2}(G)$ and $K \in \mathbb{Z}^{+|A|}$.

By summing over $K$ we conclude the proof of Theorem 2.4 for $p=2$. Other exponents will be treated in $\S 5$.

Following Stein define

$$
S_{K} f(x)=\left(\sum_{J}\left|f * \mu_{J} * \Lambda_{J}^{K}\right|^{2}\right)^{1 / 2}
$$

Proposition 4.1 follows immediately from

Lemma 4.2. There exists $\varepsilon>0$ such that

$$
\left\|S_{K} f\right\|_{2} \leq C 2^{-\varepsilon|K|}\|f\|_{2}
$$

for all $f, K$.

Fix $K$ and write $T_{J} f=f * \mu_{J} * \Lambda_{J}^{K}$. Throughout this section $\|\cdot\|_{\text {op }}$ denotes the operator norm on $L^{2}(G)$. We abuse notation slightly by writing $\|\nu\|_{1}$ to denote the total mass of any measure $\nu$. Then $\left\|T_{J}\right\|_{\text {op }} \leq C$ uniformly in $J, K$ since $\left\|\mu_{J}\right\|_{1}$ and $\left\|\Lambda_{J}^{K}\right\|_{1}$ are uniformly bounded. Lemma 4.2 results directly from the conjunction of the next two lemmas.

Lemma 4.3. There exists $\varepsilon>0$ such that $\left\|T_{J}\right\|_{\mathrm{op}} \leq C 2^{-\varepsilon|K|}$ for all $K$.

Lemma 4.4. There exists $\varepsilon>0$ such that

$$
\left\|T_{I} T_{J}^{*}\right\|_{\mathrm{op}} \leq C 2^{-\varepsilon|I-J|}
$$

for all $I, J \in \mathbb{Z}^{|A|}$ and $K \in \mathbb{Z}^{+|A|}$. 
Denote by $\tilde{\nu}$ the reflection of any measure $\nu$ about the origin:

$$
\int f d \tilde{\nu}=\int f(-x) d \nu(x)
$$

It may be assumed that all measures under discussion here are real, in which event the adjoint of an operator $T f=f * \nu$ is $T^{*} f=f * \tilde{\nu}$.

The proofs of Lemmas 4.3 and 4.4 rely on the following result, a slight extension of the main technical lemma of $[\mathrm{Cl}, \S 5]$. Let $D$ be the dimension of $\mathbf{g}$ as a vector space. Suppose that $n \geq D, \phi \in C_{0}^{\infty}\left(\mathbb{R}^{n}\right)$, and $F \in C^{\infty}$ maps a neighborhood of support $(\phi)$ to $G=\mathbb{R}^{D}$. Let $\xi$ be the push-forward $F_{*}(\phi(x) d x)$, a measure on $G$. For each $D$-tuple $E=\left(i_{1}, \ldots, i_{D}\right)$ of elements of $\{1,2, \ldots, n\}$ let

$$
J_{E}(x)=\operatorname{det}\left(\frac{\partial F_{j}}{\partial x_{i_{l}}}\right)_{1 \leq j, l \leq D}
$$

be the determinant of the Jacobian matrix, where the $\left\{F_{j}: 1 \leq j \leq D\right\}$ are the components of $F$ in our canonical coordinate system on $G$.

Lemma 4.5. Suppose that for each $z \in \operatorname{support}(\phi)$ there exists a subset $E$ and a multi-index $\gamma \in \mathbb{N}^{n}$ such that $\left(\partial^{\gamma} J_{E} / \partial x^{\gamma}(z)\right) \neq 0$. Then $\xi$ is absolutely continuous with respect to Haar measure on $G$. Moreover there exist $\varepsilon>0$ and $C_{1}<\infty$ such that for any $C_{0}<\infty$, any $\rho>0$, and any finite measure $\nu$ supported on any subset of $\left\{y \in G:|y| \leq C_{0}\right\}$ of diameter $\rho$ and satisfying $\int 1 d \nu=0$, we have

$$
\|\xi * \nu\|_{1} \leq C_{1} \rho^{\varepsilon}\|\nu\|_{1} .
$$

Another way to phrase the conclusion is that $\xi$ belongs to some Sobolev space $L_{\varepsilon}^{1+\varepsilon}, \varepsilon>0$. In the hypothesis it is not required that $\gamma$ involve only those $i_{j}$ present in $E$.

Proof. By smoothly partitioning the support of $\phi$ into finitely many regions we may reduce matters to the case where a single $E$ and $\gamma$ satisfy the hypothesis at all points in the support of $\phi$. Let $\eta>0$ be a small constant to be chosen below and partition a fixed neighborhood of the support of $\phi$ into a grid of cubes $Q$, with sides parallel to the coordinate axes, of common sidelength $\rho^{\eta}$. Construct a partition of unity $\left\{\phi_{Q}\right\}$ with each $\phi_{Q}$ supported on the cube $2 Q$ concentric with $Q$ and having twice the sidelength, satisfying $\left\|\phi_{Q}\right\|_{\infty} \leq 1$ and $\left\|\nabla \phi_{Q}\right\|_{\infty} \leq C \rho^{-\eta}$. Set

$$
\xi_{Q}=F_{*}\left(\phi_{Q} \phi d x\right)
$$

$Q$ is said to be bad if there exists $y \in 2 Q$ with $\left|J_{E}(y)\right| \leq \rho^{\eta / 3}$, and to be good otherwise. The measure of the union of the bad cubes is at most $C \rho^{\eta \delta}$, for some $\delta(\gamma)>0$, by Lemma 3.4 of [C1]. Since

$$
\left\|\xi_{Q} * \nu\right\|_{1} \leq\left\|\xi_{Q}\right\|_{1}\|\nu\|_{1} \leq C|Q| \cdot\|\nu\|_{1},
$$

we have for the contribution of the bad cubes

$$
\left\|\left(\sum_{Q \mathrm{bad}} \xi_{Q}\right) * \nu\right\|_{1} \leq C \sum_{Q \mathrm{bad}}|Q| \cdot\|\nu\|_{1} \leq C \rho^{\eta \delta}\|\nu\|_{1}
$$

as desired. 
It suffices to show that for each good $Q$,

$$
\left\|\xi_{Q} * \nu\right\|_{1} \leq C \rho^{\varepsilon}|Q| \cdot\|\nu\|_{1} .
$$

Renumber the coordinates in $\mathbb{R}^{n}$ so that $E=(1,2, \ldots, D)$. Coordinatize $\mathbb{R}^{n}$ as $\left\{(u, v) \in \mathbb{R}^{D} \times \mathbb{R}^{n-D}\right\}$. Write $Q=Q_{1} \times Q_{2}$ with $Q_{1} \subset \mathbb{R}^{D}$ and decompose

$$
\xi_{Q}=\int_{2 Q_{2}} \xi_{Q}^{v} d v
$$

where $\xi_{Q}^{v}$ is the push-forward under the map $u \mapsto F(u, v)$ of the smooth measure $\phi_{Q}(u, v) \phi(u, v) d u$ on $2 Q_{1}$. It is enough to show that

$$
\left\|\xi_{Q}^{v} * \nu\right\|_{1} \leq C \rho^{\varepsilon}\left|Q_{1}\right| \cdot\|\nu\|_{1}
$$

uniformly in $v \in 2 Q_{2}$. But since the Jacobian determinant of the function $u \mapsto f(u, v)$ is bounded below by $\rho^{\eta / 3}$ on $2 Q_{1}$ it follows from the implicit function theorem that $\xi_{Q}^{v}$ is smooth, and the discussion of equation (5.2) on p. 587 of [C1] yields the quantitative conclusion

$$
\left\|\xi_{Q}^{v} * \nu\right\|_{1} \leq C \rho^{1-C \eta}\left|Q_{1}\right| \cdot\|\nu\|_{1}
$$

for some $C<\infty$. It suffices to choose $\eta$ so that $1-C \eta>0$. The same reasoning applies to $\nu * \xi$.

Proof of Lemma 4.4. Since the $\delta_{r}$ are group automorphisms it may be arranged by a dilation which does not affect the operator norm that, for each $\alpha$, $\max \left(I_{\alpha}, J_{\alpha}\right)=0$. The operator $T_{I} T_{J}^{*}$ is given by convolution on the right with $\tilde{\Lambda}_{J}^{K} * \tilde{\mu}_{J} * \mu_{I} * \Lambda_{I}^{K}$. Assume with no loss of generality that $\min _{\alpha \in A} I_{\alpha} \leq \min _{\alpha \in A} J_{\alpha}$. Then $|I-J| \sim|I| \sim-\min _{\alpha} I_{\alpha}$, and it will suffice to show that

$$
\left\|f * \tilde{\mu}_{J} * \mu_{I} * \Lambda_{I}^{K}\right\|_{2} \leq C 2^{-\varepsilon|I|}\|f\|_{2}
$$

for all $f \in L^{2}$. Let $\xi_{0}=\tilde{\mu}_{J} * \mu_{I}$ and take $\xi$ to be the convolution product $\tilde{\xi}_{0} * \xi_{0} * \tilde{\xi}_{0} * \xi_{0} * \cdots$ with $D$ factors of $\tilde{\xi}_{0} * \xi_{0}$. As in [C1] it suffices to show that

$$
\left\|\xi * \Lambda_{I}^{K}\right\|_{1} \leq C 2^{-\varepsilon|I|}
$$

for some $\varepsilon>0$.

Assume, this time with a possible loss of generality but for the sake of simplicity, that $\min _{\alpha} I_{\alpha}=I_{\beta}$, where $\beta$ is the smallest $\alpha \in A$, in our fixed linear ordering on $A$. Let $\nu$ be the measure $\lambda_{\beta, K_{\beta}}$, dilated by $\delta_{2^{I}}$ :

$$
\int_{G} f d \nu=\int f\left(\delta_{2-I} x\right) d \lambda_{\beta, K_{\beta}}(x) .
$$

Then it is enough to show that

$$
\|\xi * \nu\|_{1} \leq C 2^{-\varepsilon|I|}
$$

in fact we will obtain a bound $C 2^{-\varepsilon|I|-\varepsilon K_{\beta}}$. Now $\|\nu\|_{1} \leq C, \nu$ annihilates constants, $\nu$ is supported in $\left\{x \in G:|x| \leq C 2^{-I_{\beta}-c K_{\beta}}\right\}$, and we shall verify that $\xi$ is a measure of the type analyzed in Lemma 4.5. 
To examine $\xi$ adopt coordinates $x=\left(x^{j}: 1 \leq j \leq D\right)$ on $\left(\mathbb{R}^{4 d}\right)^{D}$, where each $x^{j}=\left(x_{1}^{j}, \ldots, x_{4}^{j}\right) \in\left(\mathbb{R}^{d}\right)^{4}$ and in turn $x_{i}^{j}=\left(x_{i, l}^{j}: 1 \leq l \leq d\right)$ for $1 \leq i \leq 4$. Take

$$
\phi(x)=\prod_{j=1}^{D} \prod_{i=1}^{4} a\left(x_{i}^{j}\right) .
$$

Define $F: \mathbb{R}^{4 d D} \mapsto G$ by

$$
\begin{aligned}
F(x)=\prod_{j=1}^{D}[ & \exp \left(-\sum_{\alpha}\left[x_{1}^{j}\right]^{\alpha} 2^{I_{\alpha}} X_{\alpha}\right) \cdot \exp \left(\sum_{\alpha}\left[x_{2}^{j}\right]^{\alpha} 2^{J_{\alpha}} X_{\alpha}\right) \\
& \left.\cdot \exp \left(-\sum_{\alpha}\left[x_{3}^{j}\right]^{\alpha} 2^{J_{\alpha}} X_{\alpha}\right) \cdot \exp \left(\sum_{\alpha}\left[x_{4}^{j}\right]^{\alpha} 2^{I_{\alpha}} X_{\alpha}\right)\right],
\end{aligned}
$$

the product taken in order of ascending $j$ and according to the group law of $G$. $F$ depends implicitly on $I, J$; since $\max \left(I_{\alpha}, J_{\alpha}\right)=0$ for each $\alpha, F$ is $C^{\infty}$ on a fixed neighborhood of $\operatorname{support}(\phi)$, uniformly in $I, J . F$ is a homogeneous polynomial. That is, if $t x$ is defined for all $t \in \mathbb{R}$ and $x \in \mathbb{R}^{4 d D}$ by multiplying each component of $x$ by $t$, and if $F(x)$ is written as $\exp \left(\sum_{\alpha, l} Q_{\alpha, l}(x) Y_{\alpha, l}\right)$ in terms of our fixed basis for $\mathbf{g}$, then each $Q_{\alpha, l}$ is a homogeneous polynomial. This holds because the group law of $G$ and the map $\mathbb{R}^{d} \ni u \mapsto \exp \left(\sum u^{\alpha} X_{\alpha}\right)$ are homogeneous. Consequently there exists $M$ such that for each subset $E$ of $\{\zeta=(j, i, \alpha): j \in\{1,2, \ldots, d\}, i \in\{1,2,3,4\}, \alpha \in A\}$ with $|E|=D$, the Jacobian determinant

$$
J_{E}=\operatorname{det}\left(\frac{\partial F}{\partial x_{\zeta}}\right)_{\zeta \in E}
$$

is a homogeneous polynomial of degree $M$, or is identically zero. Here $x_{\zeta}$ denotes $x_{i, \alpha}^{j}$.

Lemma 4.6. There exists $E$ such that $J_{E}$ does not vanish identically.

Consequently for this $E$ there exists $\tau$ with $|\tau|=M$ such that $\partial^{\tau} J_{E} / \partial x^{\tau}$ is a nonzero constant which may depend on $I, J$. This lemma is a direct consequence of the next result, proved implicitly in [C1] (see the proof there that the three formulations of " $\gamma$ generates $G$ " are equivalent). The argument will not be repeated here. In the following statement $J_{E}$ denotes the Jacobian determinant defined as above, but with $F$ replaced by $\Phi$.

Lemma 4.7. Let $\mathbf{h}$ be a nilpotent Lie algebra and $H$ the associated connected, simply connected nilpotent group. Let $D$ be the dimension of $h$ as a vector space. Suppose that $n$ is a positive integer, $B$ a finite subset of $\mathbb{N}^{n} \backslash\{0\}$, and $\left\{X_{\beta}: \beta \in B\right\}$ is a subset of $\mathbf{h}$ which generates it as a Lie algebra. Define $\Phi:\left(\mathbb{R}^{n}\right)^{D} \mapsto H$ by

$$
\Phi\left(y_{1}, \ldots, y_{D}\right)=\prod_{j=1}^{D} \exp \left(\sum_{\beta} y_{j}^{\beta} X_{\beta}\right) .
$$

Then at least one of the Jacobian determinants $J_{E}$ is not identically zero. 
Now the measure $\xi=\tilde{\mu}_{I} * \mu_{J} * \tilde{\mu}_{J} * \mu_{I} * \cdots$ is nothing but $F_{*}(\phi(x) d x)$, so $\xi$ and $\nu$ satisfy the hypotheses of Lemma 4.4, with $\rho \leq C 2^{-|I|-c K_{\beta}}$, whence for each $I, J$ there exists $C_{I, J}$ such that

$$
\|\xi * \nu\|_{1} \leq C_{I, J} 2^{-\varepsilon|I|-\varepsilon K_{\beta}}
$$

as desired. $\varepsilon$ is independent of $I, J$ since there are only finitely many possible values of $E$ and $\gamma$. However we need to know that $C_{I, J}$ may also be taken to be independent of $I, J$. This follows from compactness considerations. Permit each component $I_{\alpha}$ and $J_{\alpha}$ to take on the value $-\infty$, always with the stipulation that $\max \left(I_{\alpha}, J_{\alpha}\right)=0$ for each $\alpha$. The convention is of course that $2^{-\infty}=0$ in the definition of $F$. Then $F$ is still $C^{\infty}$ uniformly in $I, J$. Lemma 4.6 remains valid with the same proof, hence $C_{I, J}$ is uniformly bounded by compactness.

We have assumed that $I_{\beta}=\min \left\{I_{\alpha}\right\}$ occurs when $\beta$ is the smallest of the $\alpha$. In the general case it suffices to show that

$$
\left\|\xi * \prod_{\alpha \leq \beta} \lambda_{\alpha, K_{\alpha, I}}\right\|_{1} \leq C 2^{-\varepsilon I_{\beta}},
$$

where now

$$
\int f d \lambda_{\alpha, K_{\alpha, I}}=\int f\left(\delta_{r} x\right) d \lambda_{\alpha, K_{\alpha}}(x), \quad r=2^{-I} .
$$

The factor $\xi * \prod_{\alpha<\beta} \lambda_{\alpha, K_{\alpha, I}}$ has the same properties as does $\xi$ for our purposes, uniformly in $I, K$; indeed so does the convolution of $\xi$ with any finite measure supported in a fixed compact region. $\nu$ should now be taken to be $\lambda_{\beta, K_{\beta, I}}$ and Lemma 4.4 again applies.

The same arguments establish Lemma 4.3. By homogeneity matters reduce to the case $J=0$. Then $\xi$ should be taken to be the convolution product of factors $\mu_{0}$ and $\tilde{\mu}_{0}$, convolved with $\prod_{\alpha<\beta} \lambda_{\alpha, K_{\alpha}}$, where $K_{\beta}=\max \left\{K_{\alpha}\right\}$, and $\nu$ should be taken to be $\lambda_{\beta, K_{\beta}}$.

\section{EXPONENTS $p \neq 2$}

Lemma 5.1. For each $p>1$ there exists $C<\infty$ such that the $L^{p}$ operator norm of $f \mapsto \sup _{J}\left|f * \mu_{J} * \Lambda_{J}^{K}\right|$ is majorized by $C(1+|K|)^{D}$.

Since the $L^{2}$ operator norm is at most $C 2^{-\varepsilon|K|}$ we may interpolate to find that for each $p \in(1, \infty)$ there exists $\eta>0$ such that the $L^{p}$ operator norm is actually no worse than $C 2^{-\eta|K|}$. Summation over all $K \in \mathbb{Z}^{+}$completes the proof of Theorem 2.4 .

To prove the lemma recall a variant of the Hardy-Littlewood maximal function on $\mathbb{R}^{1}$. For $0<\tau \leq 1$ and some $y_{0}$ satisfying $\left|y_{0}\right| \leq 10$ define

$$
M f(x)=\tau^{-1} \sup _{j \in \mathbb{Z}} \int_{\left|y-y_{0}\right| \leq \tau}\left|f\left(x-2^{-j} y\right)\right| d y .
$$

Then $M$ is bounded on $L^{p}(\mathbb{R})$ for all $p>1$, with bound $C_{p} \log \left(1+\tau^{-1}\right)$; see for instance [S]. Combining this result with the iteration and majorization argument sketched in the introduction and employed in the proof of Lemma 3.2 gives 
Lemma 5.2. For each $p>1$ there exists $C_{p}<\infty$ such that for all $\tau \in(0,1]$, all Euclidean balls $B$ contained in a fixed compact region of $G$, and all $f \in L^{p}(G)$,

$$
\left\|\sup _{J}|B|^{-1} \int_{B}\left|f\left(x \cdot\left[\delta_{2^{-\jmath}} y\right]^{-1}\right)\right| d y\right\|_{p} \leq C_{p}\left[\log \left(1+|B|^{-1}\right)\right]^{D}\|f\|_{p} .
$$

The measure $\Lambda_{0}^{K}$ is absolutely continuous and in fact has a $C^{\infty}$ RadonNikodym derivative with respect to Haar measure, for all $K \in \mathbb{Z}^{+}$. Applying Lemma 5.2 in a straightforward way results in Lemma 5.1.

Since this paper was completed, interesting results concerning analogous singular integral operators, in the abelian case, have been obtained by Ricci and Stein.

\section{REFERENCES}

[B] J. Bourgain, Averages in the plane over convex curves and maximal operators, J. Analyse Math. 47 (1986), 69-85.

[CSS] H. Carlsson, P. Sjögren, and J. Strömberg, Multi-parameter maximal functions along dilation-invariant hypersurfaces, Trans. Amer. Math. Soc. 292 (1985), 335-343.

[C1] M. Christ, Hilbert transforms along curves, I. Nilpotent groups, Ann. of Math. (2) 122 (1985), 575-596.

[C2] - Hilbert transforms along curves, III. Rotational curvature, preprint, December 1984, unpublished.

[JMZ] B. Jessen, J. Marcinkiewicz, and A. Zygmund, Note on the differentiability of multiple integrals, Fund. Math. 25 (1935), 217-234.

[PS1] D. H. Phong and E. M. Stein, Singular integrals related to the Radon transform and boundary value problems, Proc. Nat. Acad. Sci. U.S.A. 80 (1983), 7697-7701.

[PS2] _ Hilbert integrals, singular integrals and Radon transforms, Acta Math. 157 (1987), 99-157.

[RSj1] F. Ricci and P. Sjögren, Multiparameter maximal functions along dilation-invariant hypersurfaces, Trans. Amer. Math. Soc. 292 (1985), 335-343.

[RSj2] F. Ricci and P. Sjögren, Two-parameter maximal functions on the Heisenberg group, preprint.

[RiS1] F. Ricci and E. M. Stein, Oscillatory singular integrals and harmonic analysis on nilpotent groups, Proc. Nat. Acad. Sci. U.S.A. 83 (1986), 1-3.

[RiS2] _ Harmonic analysis on nilpotent groups and singular integrals. II: Singular kernels supported on submanifolds, J. Funct. Anal. 78 (1988), 56-84.

[RoS] L. P. Rothschild and E. M. Stein, Hypoelliptic differential operators and nilpotent groups, Acta Math. 137 (1976), 247-320.

[S] E. M. Stein, Boundary behavior of harmonic functions on symmetric spaces: Maximal estimates for Poisson integrals, Invent. Math. 74 (1983), 63-83.

[SW] E. M. Stein and S. Wainger, Problems in harmonic analysis related to curvature, Bull. Amer. Math. Soc. 84 (1978), 1239-1295. 